\title{
LA COMUNICACIÓN COMO ESTRATEGIA DE DESARROLLO Y ORGANIZACIONAL EN LA GESTIÓN DEL CONOCIMIENTO
}

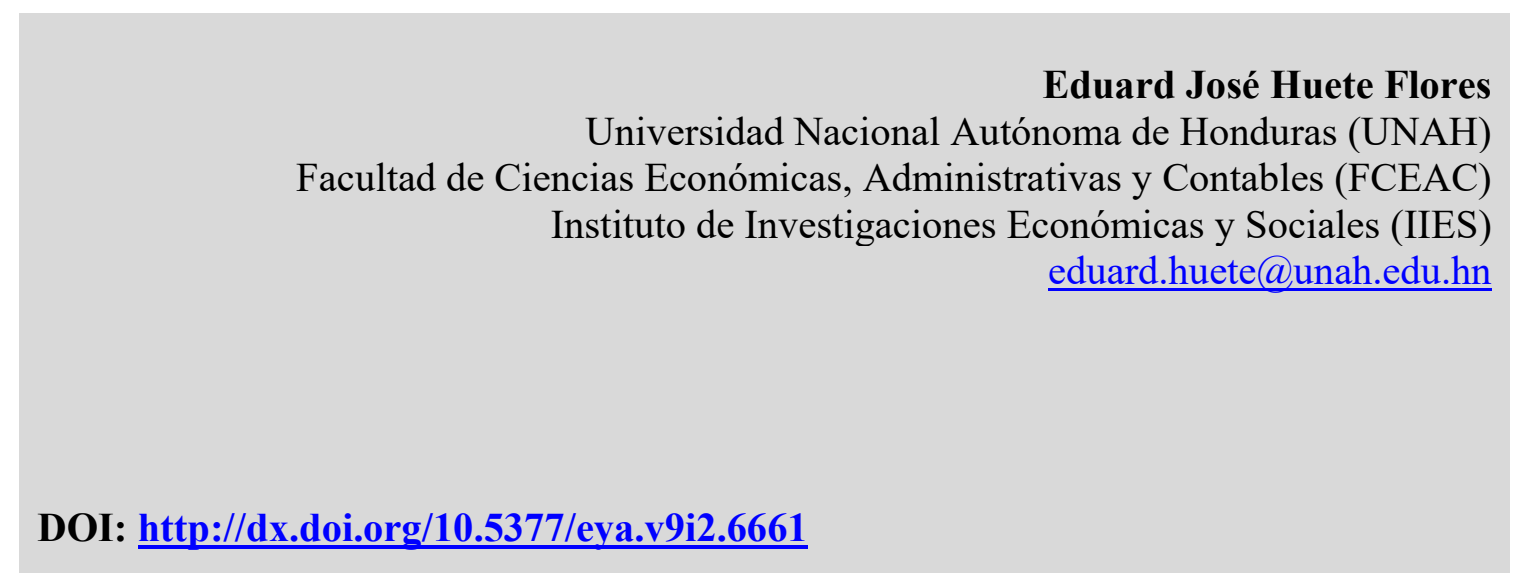

\section{RESUMEN}

La gestión del conocimiento juega un papel trascendental en las economías del conocimiento, siendo a través de procesos metodológicos la inserción de las organizaciones a una nueva etapa productiva, la cual no solo es medida por su infraestructura o el recurso financiero que dispone, si no, considerando su recurso humano y su conocimiento individual como el factor de mayor trascendencia para introducirse en una nueva era evolutiva. La investigación es de tipo descriptiva, la cual ilustra el proceso que deben incorporar las empresas en sus culturas organizacionales para empezar en la gestión del conocimiento.

Palabras claves: Gestión del
conocimiento, La Comunicación,
Estrategias




\title{
COMMUNICATION AS A DEVELOPMENT AND ORGANIZATIONAL STRATEGY IN THE MANAGEMENT OF KNOWLEDGE
}

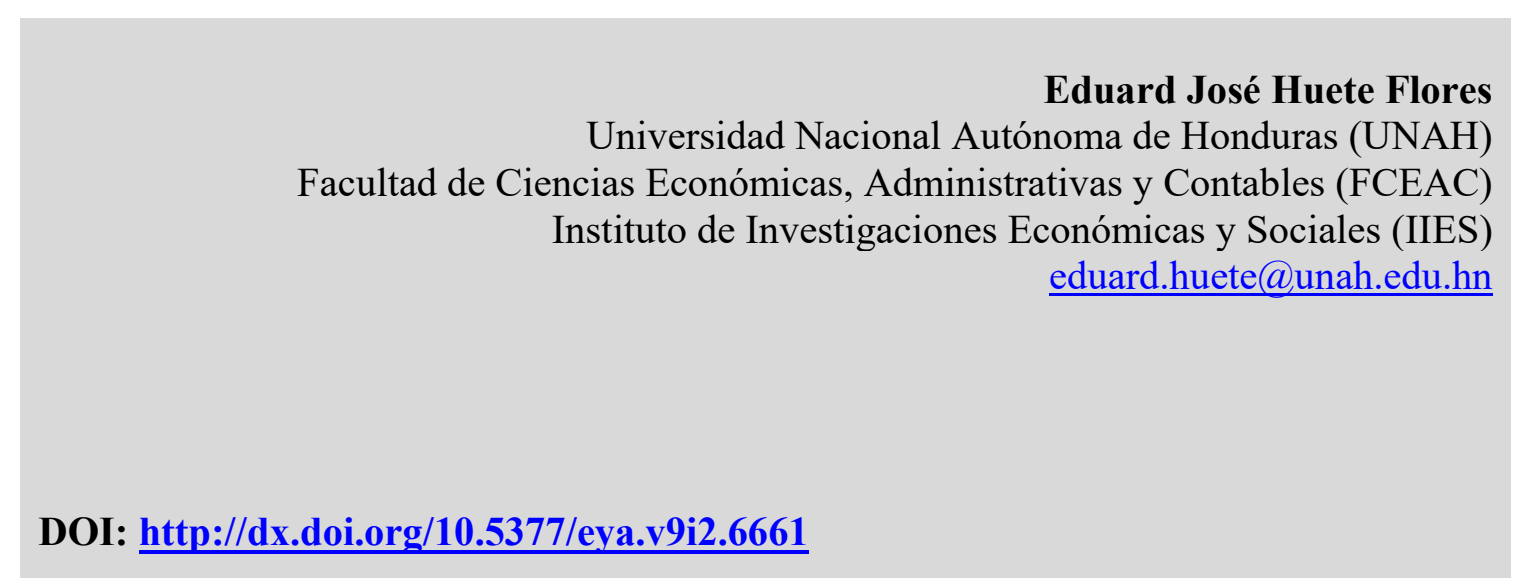

\begin{abstract}
Knowledge management plays a transcendental role in the knowledge economies, being through methodological processes the insertion of the organizations to a new productive stage, which is not only measured by its infrastructure or the financial resource available, if not, considering their human resources and their individual knowledge as the most important factor to enter a new evolutionary era. The research is descriptive, which illustrates the process that companies must incorporate into their organizational cultures to start in knowledge management.
\end{abstract}

Keywords: Knowledge management, Communication, Strategies 


\section{LA COMUNICACIÓN COMO ESTRATEGIA DE DESARROLLO Y ORGANIZACIONAL EN}

LA GESTIÓN DEL CONOCIMIENTO

Revista Economía y Administración, Vol. 9, No. 22018

ISSN 2219-6722 / ISSNE 2222-2707

\section{INTRODUCCIÓN}

La actual investigación, tiene como propósito difundir las estrategias $\mathrm{y}$ modelos que deben incorporarse en una organización para introducirse a la nueva era de la gestión del conocimiento, factor que forma parte de la economía del conocimiento. Encontrando que la comunicación es la herramienta esencial a primera instancia para introducirse en la era del conocimiento, herramienta que explora los conocimientos existentes pero que de manera simultánea descubre los nuevos, recolectando la información idónea a través de un modelo que debe aplicarse hacia los colaboradores que integran la organización. A la vez, se da a conocer la estrategia que socializa y difunde los nuevos conocimientos para luego ser implementado el nuevo conocimiento dentro de la organización, considerando oportuno la innovación y el cambio tecnológico dentro de la misma.

\section{MARCO CONCEPTUAL}

\subsection{Activos del conocimiento}

Nieves (2015, pág. 32) plantea el conocimiento desde diferentes enfoques, considerándolos como activos del conocimiento, dividiéndolos desde cuatro (4) esferas: un elemento complejo, como factor que no expresa la realidad, si no que percibe la acción de esa realidad. Privativo de las personas, cada individuo posee conocimiento inherente, el cual debe compartirse para crear innovación. Un
Proceso, refiriéndose a la interacción social. Una Verdad, determina lo que realmente funciona y descarta lo que no. De igual manera, Nieves plantea un esquema de como se puede visualizar esas esferas dentro del conocimiento (ver Figura $\mathrm{N}^{\circ} 1$ ).

Figura No. 1 Abordaje del Conocimiento

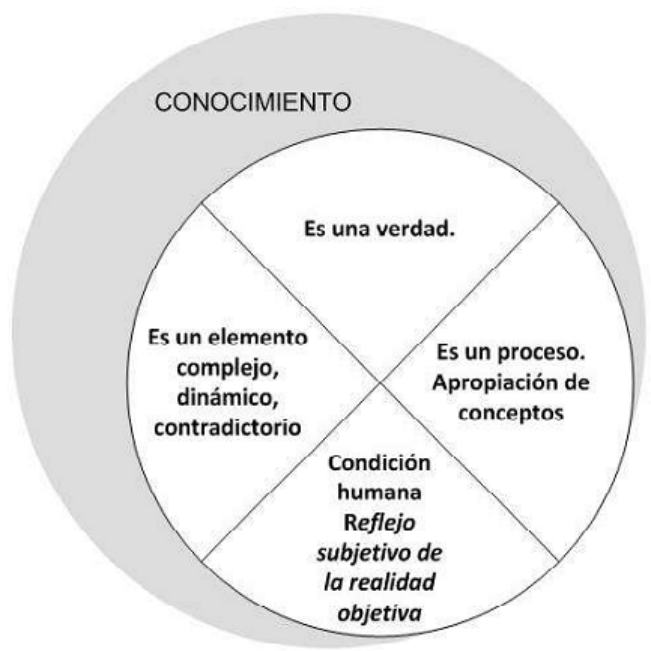

Fuente: Nieves, 2015

Por otra parte, Mejia y Colín (2013, pág. 28), describen las características del conocimiento, planteando lo siguiente:

a) Es tácito, debido a que cada significado de este es vulnerable a las experiencias particulares de los individuos.

b) Es dinámico, capaz de generar nuevos conocimientos, transformarlos y perfeccionarlos.

c) Es delimitado, debido a las limitaciones del cerebro humano. 


\section{LA COMUNICACIÓN COMO ESTRATEGIA DE DESARROLLO Y ORGANIZACIONAL EN}

LA GESTIÓN DEL CONOCIMIENTO

Revista Economía y Administración, Vol. 9, No. 22018

ISSN 2219-6722 / ISSNE 2222-2707

d) Es movible, transferible para otras personas.

\subsection{Economía basada en el conocimiento}

Según la CEPAL, en su informe titulado Introducción a la gestión del conocimiento y su aplicación al sector público (2002, pág. 8), menciona que "los síntomas iniciales de la llamada Nueva Economía aparecen en la década de los sesenta, especialmente con el surgimiento de los primeros computadores, la expansión de las multinacionales y la formación de un mercado internacional de capitales." Continúa describiendo el avance de la economía del conocimiento durante la década de los ochentas determinando serios cambios mundiales que cambiaron la forma de organizar procesos como ser:

- El cambio a organizaciones más flexible.

- Cambios en las empresas japonesas.

- La consolidación del mercado internacional.

- Las innovaciones en materia de tecnologías.

Esta serie de cambios contribuyeron a la búsqueda de alternativas para dinamizar los mercados internacionales lo cual refleja la importancia del conocimiento para afrontar las crisis mundiales.

\footnotetext{
${ }^{8}$ Economista y profesor estadounidense. Ha recibido la Medalla John Bates Clark y el Premio Nobel de Economía
}

\subsubsection{Características del conocimiento en la economía}

De igual manera, el informe de la CEPAL (2002, pág. 10), plantea una serie de teóricos quienes describen características de la economía, basadas en el conocimiento. Conceptualizando la economía como "un sistema en el cual el motor de creación de valor y/o beneficios es el conocimiento y la capacidad para construirlo por medio del aprendizaje (A, 2002)." También, “el conocimiento siempre ha sido reconocido como factor clave en la sociedad ......, este no pasó a tener un significado económico como lo tiene en la actualidad (Dahlman \& Aubert, 2001). Pero mantener el conocimiento y contar con herramientas tecnológicas no es suficiente. Por ello, "lo importante es poseer competencias claves como la capacidad de aprender, de incorporar conocimiento nuevo y de dar nuevas respuestas, algunos autores la han llamado la "Sociedad del Aprendizaje del Conocimiento Social (Didriksson, 2003)”.

\subsubsection{Bases del Conocimiento}

Diversos autores han planteado las bases del conocimiento, dentro de una economía del conocimiento. Uno de ellos es Stiglitz ${ }^{8}$ quien conceptuó el conocimiento y su gestión en el desarrollar sinergias dentro del sistema y la dinámica manifestándolo 
Revista Economía y Administración, Vol. 9, No. 22018

ISSN 2219-6722 / ISSNE 2222-2707

a través de cuatro aspectos fundamentales (Peluffo \& Catalán, 2002, págs. 10,11):

- Importancia del conocimiento como factor de crecimiento y de progreso: la importancia de la educación como clave del desarrollo.

- Desarrollo de procesos de apropiación social del conocimiento: refiriéndolo como un bien común que permite que las organizaciones o instituciones se interrelacionen con él.

- La capacidad de generar procesos dinámicos de aprendizaje social: lo relaciono con el fortalecimiento de las competencias en las personas.

- La Gestión Estratégica del Conocimiento por medio de un pensamiento estratégico $\mathrm{y}$ prospectivo: generación del conocimiento y del cambio social como elementos de desarrollo sustentable.

De igual manera, el informe de la CEPAL (2002, pág. 11) menciona "no solo hay que desarrollar alta tecnología, sino también aumentar la capacidad de las personas e instituciones en la adquisición, generación, difusión y uso del conocimiento más efectivamente para producir desarrollo social y crecimiento económico."

\subsection{Gestión del conocimiento}

Para Sánchez (2005), la gestión del conocimiento es el conjunto de procesos y sistemas que hacen que el capital intelectual de la organización crezca. Para gestionar el capital intelectual es necesaria la gestión del conocimiento en sus dos dimensiones:

Hard: Aspectos más duros o formalizables. Dentro de esta, se encuentran aquellas incluidas en el capital intelectual con posible cuantificación: el capital estructural y el capital relacional.

Soft: Aspectos más blandos o no formalizables. Dentro de esta, se encuentra la variable fundamental del capital intelectual: el capital humano, es decir, los conocimientos atesorados en los cerebros de los empleados producto del aprendizaje.

De igual manera Sánchez (2005) referencia a Nonaka y Takeuchi, quienes sostuvieron que el conocimiento en las organizaciones surge de las personas, pero es en el proceso de compartirlo entre los colaboradores es donde genera un conocimiento valioso para la organización como un todo. Establecieron patrones los cuales se describen a continuación:

- De tácito a tácito, que se produce cuando los individuos comparten conocimiento mediante observación, imitación y/o práctica.

- De explícito a explícito, que se produce cuando un individuo combina elementos del conocimiento existente, adaptando o mostrando una nueva perspectiva. 


\section{LA COMUNICACIÓN COMO ESTRATEGIA DE DESARROLLO Y ORGANIZACIONAL EN}

LA GESTIÓN DEL CONOCIMIENTO

Revista Economía y Administración, Vol. 9, No. 22018

ISSN 2219-6722 / ISSNE 2222-2707

- De tácito a explícito, que se produce cuando un individuo es capaz de articular los fundamentos de su conocimiento tácito, lo convierte en explícito y se logra por consiguiente un crecimiento de la base de conocimiento.

- De explícito a tácito, que se produce cuando el conocimiento explícito es compartido a lo largo de la organización, los demás comienzan a utilizarlo y por lo tanto lo amplían, extienden e incorporan en su propio conocimiento tácito.

\subsubsection{La Comunicación como estrategia en la gestión del conocimiento}

Garcia Jimenez (2002), describe que "Las empresas inteligentes adquieren mayor sentido en una correcta gestión de la información; pero hoy se exige no sólo una eficaz gestión de la información, sino que esta se ha de convertir en conocimiento". Asigna una gran importancia a la gestión de la información, la cual implícitamente se encuentra la comunicación.

De igual forma Irene Martín (2007, pág. 54) describe que los responsables de comunicación son a primera instancia los que facilitaran los recursos para acceder a la información por medio de tecnología $u$ otros métodos que puedan adaptarse en

\footnotetext{
${ }^{9}$ Filosofía Industrial de eliminación de todo lo que implique desperdicio en el proceso de
}

cada organización. El propósito de este accionar es que el conocimiento pueda generalizarse y pueda ser transferido para la solución de todos los problemas que forman parte del funcionamiento de la organización como afirman Cayuela y Guerra. También, Martín menciona y referencia a Garcia y Val Núñez quienes indican que una organización que aprende es una organización que gira en torno a los equipos de trabajo, aprovecha el recurso humano, mantiene una cultura flexible, innovadora e integrada. De igual manera sobresalen aspectos como el just in time ${ }^{9}$.

Aspecto clave es la evolución cultural en las organizaciones según lo menciona Martin (2007, pág. 54) en la que debe transformarse su cultura basándose en el conocimiento superando los obstáculos y enfocándose en la generación, difusión, transferencia y socialización de información y el conocimiento. Superar los problemas no es tarea fácil a continuación describe algunos aspectos a tener en claro:

- Dificultad para la evolución de la cultura interna. Esto impide el cambio de comportamientos; por lo tanto, genera incapacidad para participar, compartir, colaborar.

- Dificultad individual para la identificación de la información relevante.

producción, desde las compras hasta la distribución. 
Revista Economía y Administración, Vol. 9, No. 22018

ISSN 2219-6722 / ISSNE 2222-2707

- Carencias individuales y grupales de conocimiento y brechas de competencias organizacionales, lo que impide la creación de conocimiento organizacional mediante el aprendizaje.

- Dificultad para considerar el aprendizaje organizacional como estrategia.

- Dificultad en la participación para la creación de nuevo conocimiento.

- Falta de vías para optimizar los tiempos de captación de competencias.

- Falta de espacios, canales y herramientas adecuados para almacenar, distribuir y permitir el acceso al conocimiento.

- Respecto a la primera y más importante de las barreras, la relativa al cambio cultural requerido, la comunicación deberá ejercer su función como formadora de la nueva cultura organizativa

\subsubsection{Identificación de la información relevante}

Para lograr la identificación de la información es vital examinar los enfoques

\footnotetext{
${ }^{10}$ Es un sistema de información que va más allá de las barreras de una organización, incluyendo de manera temporalmente indefinida a diversas empresas, con el fin de satisfacer las necesidades estratégicas de éstas
}

estratégicos basado en el conocimiento, Martín (2007) identifica la generación y aplicación como los pilares fundamentales de la empresa, según afirman autores como Kogut y Zander (1993), Nonaka y Takeuchi (1995) o Foss (1996). Este proceso tiene lugar dentro de la comunidad de interacción de la empresa, que atraviesa niveles interorganizativos ${ }^{10} \quad \mathrm{e}$ intraorganizativos. $^{11}$

Otra forma que expresa Martín (2007) es cuando las empresas comparten conocimientos por medio de alianzas y la colaboración con otras organizaciones. La experiencia laboral de las personas resulta valiosa, porque ofrece la oportunidad de analizar y planificar capacidades. $\mathrm{Su}$ aprovechamiento permite que la empresa las transforme en conocimientos estructurados, por medio del análisis y la realimentación. La generación del conocimiento comprende tres actividades:

- La creación interna de conocimiento, que se obtiene de la investigación y diseño dentro de la empresa.

- El aprendizaje por acción, que consiste en el entrenamiento en el trabajo, experimentos $\mathrm{y}$ simulaciones.

- La adquisición y el acceso del conocimiento externo (licencias,

\footnotetext{
11 Sistema que se refiere a la gestión de la información interna (programas informáticos de contabilidad, etc.), interacción electrónica de diversas organizaciones independientes y otros medios de comunicación con el entorno.
} 


\section{LA COMUNICACIÓN COMO ESTRATEGIA DE DESARROLLO Y ORGANIZACIONAL EN \\ LA GESTIÓN DEL CONOCIMIENTO}

Revista Economía y Administración, Vol. 9, No. 22018

ISSN 2219-6722 / ISSNE 2222-2707

\begin{abstract}
subcontrataciones, alianzas
estratégicas, proveedores, clientes, universidades, laboratorios de investigación, inventores independientes, agencias gubernamentales, implementación de tecnología y de sistemas de información).
\end{abstract}

Para satisfacer a los stakeholders es preciso transformar y poner en marcha el conocimiento generado por cada organización según Martín (2007, pág. 59). Menciona que existen tres procesos claves para crear conocimiento organizacional, siendo la comunicación la herramienta eficaz para poder realizarlo como estrategia, planeando lo siguiente:

- En primer lugar, la información debe ser de elevada calidad, a fin de que pueda transformarse en conocimiento organizativo.

- En segundo término, la experiencia y los conocimientos tácitos de los empleados tienen que salir a la luz.

- Por último, es prioritario obtener y compartir los conocimientos relativos al know-what ${ }^{12}$, knowhow $^{13}$ y know-why ${ }^{14}$. (Figura $\mathrm{N}^{\circ} 2$ )

\footnotetext{
${ }^{12}$ Conocimiento personal o de un equipo

${ }^{13}$ Experiencia de una persona en su campo, sabe realizar una tarea debido a que lleva mucho tiempo haciéndola.

${ }^{14}$ Este conocimiento permite a las personas dar un paso más allá de los conocimientos técnicos y
}

Figura 2 - Proceso de creación del conocimiento organizativo

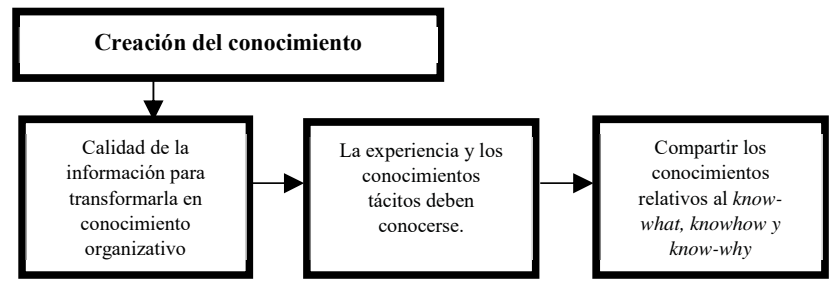

\subsection{EI Aprendizaje}

Irene Martin (2007, pág. 60), conceptualiza el aprendizaje desde diferentes ópticas como ser:

Es un proceso de construcción de conocimiento. El aprendizaje es dependiente del conocimiento; las personas usan su conocimiento para crear nuevo conocimiento. El aprendizaje necesita distribución cognitiva. Esto implica combinar el conocimiento que se posee con el conocimiento exterior.

\subsubsection{Estrategias para plasmar el conocimiento}

En el artículo Retos de la comunicación corporativa en la sociedad del conocimiento: de la gestión de información a la creación de conocimiento organizacional (Martín, 2007, pág. 61) plantea estrategias para gestionar el conocimiento explicito. En la figura No.3 se ilustra el proceso en el cual, las personas

crear extraordinario empuje por medio del conocimiento, incluyendo la capacidad para hacer frente a las interacciones desconocidas y situaciones que no son evidentes 


\section{LA COMUNICACIÓN COMO ESTRATEGIA DE DESARROLLO Y ORGANIZACIONAL EN \\ LA GESTIÓN DEL CONOCIMIENTO}

Revista Economía y Administración, Vol. 9, No. 22018

ISSN 2219-6722 / ISSNE 2222-2707

poseedoras del conocimiento puedan plasmarlo en documentos. mientras que en la figura No. 4 se refiere al conocimiento tácito, el cual muestra una comunicación directa entre personas en el cual comparten el conocimiento a un individuo y este se encarga de plasmarlo en un documento, este proceso se realiza para mantener una calidad de la extracción de conocimiento y es una forma práctica de sintetizar y codificar el conocimiento organizacional en la jerarquía baja de la organizacional.

Con las estrategias planteadas se busca gestionar el conocimiento organizacional, el cual extraiga la calidad y experiencia de sus colaboradores en documentos que luego serán implementados como manuales de puestos dentro de la organización. Lo que significaría una correcta funcionalidad y operacionalidad de las actividades organizacionales por medio del recurso intangible de sus colaboradores.

Figura No. 3: Estrategia para plasmar el conocimiento explícito.

Estrategia del Conocimiento Explícito

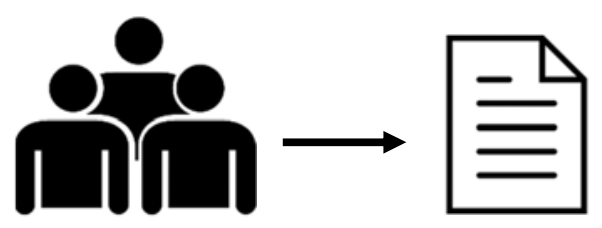

Figura No. 4: Estrategia para plasmar el conocimiento tácito

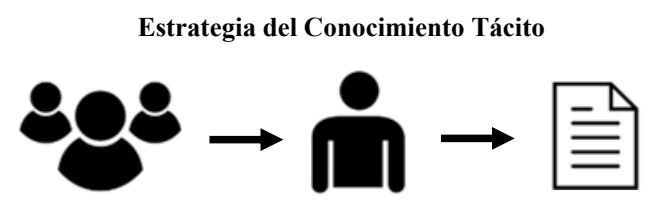

Manteniendo lo expuesto por Martin (2007, pág. 61) describe el modelo planteado por Leem de como extraer y aprovechar el conocimiento de los colaboradores. En la figura 5 se visualiza el proceso a realizar. Propone comenzar con el cambio de cultura en la organización y como se describió anteriormente debe estar orientada a la gestión del conocimiento, luego buscar los mecanismos para transformar el conocimiento tácito en explicito. De igual manera es generar y compartir el conocimiento organizacional, luego evaluar las prácticas y mejorarlas, finalizando con el fomento de una organización con la generación y el compartir del conocimiento. 


\section{LA COMUNICACIÓN COMO ESTRATEGIA DE DESARROLLO Y ORGANIZACIONAL EN \\ LA GESTIÓN DEL CONOCIMIENTO}

Revista Economía y Administración, Vol. 9, No. 22018

ISSN 2219-6722 / ISSNE 2222-2707

Figura 5 Modelo para el aprovechamiento de la información

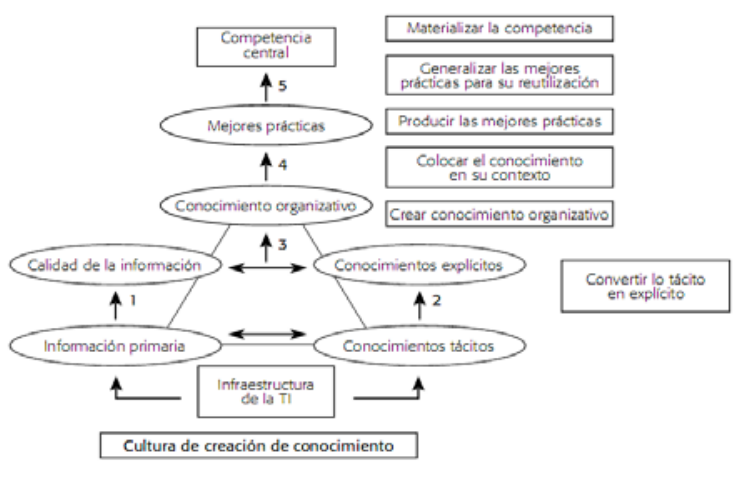

Fuente: Leem (1997).

\subsubsection{Estrategia de construcción y socialización del conocimiento}

Para construir y socializar el conocimiento Irene Martin (2007, pág. 62) plantea estrategias que permitan mantener una cultura del conocimiento en las organizaciones:

Estructurar procesos y canales de comunicación necesarios para gestionar la información, construir y socializar conocimiento. Compartir el conocimiento individual para transformarlo en conocimiento social. Flexibilizar la organización de forma que todos sus miembros se sientan motivados a proponer, innovar y colaborar en la "dinamización" de la organización. Hacer a la organización más sensible a las contribuciones de sus miembros y a los cambios que se propongan a partir de las nuevas necesidades o el aprendizaje organizacional.

\section{METODOLOGÍA}

La presente investigación es de tipo descriptivo, la cual provee las teorías relevantes para la recolección de información a través de modelos, los cuales son aplicados hacia los colaboradores de la organización, haciendo uso de la herramienta de la comunicación, como factor prioritario para introducirse en la gestión del conocimiento organizacional.

\section{CONCLUSIÓN}

La comunicación es uno de los factores prioritarios para introducirse en la gestión del conocimiento organizacional, ya que a través de un cambio en la cultura e implementar los modelos para recolectar información relevante por parte de los colaboradores, será el elemento clave para involucrarse en la economía del conocimiento, ya que el recurso humano y su conocimiento es esencial para formar parte de esta economía.

Introducirse a la comunidad organizacional gestora del conocimiento, conlleva un proceso de alto costo, ya que no solo involucra extraer el conocimiento de los colaboradores, si no la aplicación en sus procesos, los cuales deben de realizarse con la incorporación de métodos que forman parte de las Tecnologías de la Información y la Comunicación (TIC), las cuales inciden de manera circunstancial en la inversión tecnológica en las organizaciones. Por consiguiente, las organizaciones deben crear un plan 


\section{LA COMUNICACIÓN COMO ESTRATEGIA DE DESARROLLO Y ORGANIZACIONAL EN \\ LA GESTIÓN DEL CONOCIMIENTO}

Revista Economía y Administración, Vol. 9, No. 22018

ISSN 2219-6722 / ISSNE 2222-2707

gerencial a largo plazo, el cual, los guie paso a paso para formar parte de las organizaciones que integran las economías del conocimiento.

\section{REFERENCIAS}

A, M. (2002). Introducción a la gestión del conocimiento y su aplicación al sector público: Naciones Unidas, CEPAL, ILPES. Recuperado de https://books.google.hn/books?id=VP 8kawCabhkC

Chaparro, F. (2001). Conocimiento, aprendizaje y capital social como motor de desarrollo. Ciência da Informação, 30(1), 19-31. doi:10.1590/S010019652001000100004

Choo, C. W., \& Rey Díaz, D. (1999). La organización inteligente: El empleo de la información para dar significado, crear conocimiento $y$ tomar decisiones. México: Oxford University Press.

Dahlman, C. J., \& Aubert, J.-E. (2001). China and the Knowledge Economy: The World Bank.

Davenport, T. H., \& Prusak, L. (2001). Conocimiento en acción: cómo las organizaciones manejan lo que saben: Pearson Educación. Recuperado de https://books.google.hn/books?id=Zlr vAQAACAAJ

Didriksson, A. (2003). La sociedad del conocimiento desde la perspectiva latinoamericana. Memorias Del IV Encuentro de Estudios Prospectivos Región Andina: Sociedad, Educación y Desarrollo: Medellín,

García Jiménez, A. (2002). Organización y gestión del conocimiento en la comunicación. Biblioteconomía y admnistración cultural: Vol. 64. [Gijón]: Trea.

Gil, M. (2003). Universidad, sector productivo y sustentabilidad: Universidad Central de Venezuela, Consejo de Desarrollo Científico y Humanístico. Recuperado de

https://books.google.hn/books?id=Cn IIzDmIAQcC

Grant, R. M., Fernández, Z., Lorenzo Gómez, J. D., \& Ruiz Navarro, J. (1996). Dirección estratégica: Conceptos, técnicas y aplicaciones. Biblioteca Civitas Economía y Empresa. Colección Empresa. Tratados y Manuales. Madrid: Civitas.

Martín, I. (2007). Retos de la comunicación corporativa en la sociedad del conocimiento: de la gestión de información a la creación de conocimiento organizacional. Signo $y$ Pensamiento, XXVI(51), 52-67. Recuperado de http://www.redalyc.org/pdf/860/8600 5106.pdf

Mejía, M., \& Colín, M. (2013). Gestióbn del conocimiento y su importancia en las organizaciones. Revista TRILOGÍA, 25-35. Obtenido de http://web.a.ebscohost.com/ehost/pdf viewer $/$ pdfviewer? vid $=5 \&$ sid $=872 \mathrm{c} 9$ bc1-e8ce-4a08-a0bcb005208c1bd8\%40sessionmgr4006

Nieves, Y. (2015). Activos de aprendizajer organizacional un activo de conocimiento critico . Ciencias de la Información , 31 - 37. Obtenido de https://www.redalyc.org/pdf/1814/18 1443340005.pdf

Passaillaigue, R., \& Estrada, V. (2016). La gestión del conocimiento y el aprendizaje organizacional en 


\section{LA COMUNICACIÓN COMO ESTRATEGIA DE DESARROLLO Y ORGANIZACIONAL EN \\ LA GESTIÓN DEL CONOCIMIENTO}

Revista Economía y Administración, Vol. 9, No. 22018

ISSN 2219-6722 / ISSNE 2222-2707

instituciones de educación superior.

GECONTEC: Revista Internacional de Gestión del Conocimiento y la Tecnología, 35-43.

Peluffo, M., \& Catalán, E. (2002). Introducción a la gestión del conocimiento y su aplicación al sector publico. Santiago: Comisión Económica para América Latina y el Caribe (CEPAL). Obtenido de http://unpan1.un.org/intradoc/groups/ public/documents/uneclac/unpan0145 65.pdf

Pulido-Suárez, M., Ortiz-Zamora, A., \& Uribe, L. (2017). Las organizaciones en la economía del conocimiento:El papel fundamental de la gestión y transferencia del conocimiento en las organizaciones actuales. UniPluri/Versidad, 77-86. Obtenido de http://web.a.ebscohost.com/ehost/pdf viewer/pdfviewer? vid $=3 \&$ sid $=872 \mathrm{c} 9$ bc1-e8ce-4a08-a0bc-

b005208c1bd8\%40sessionmgr4006

Rodríguez-Ponce, E., Pedraja-Rejas, L., Delgado, M., \& Rodríguez-Ponce, J. (2010). GESTIÓN DEL CONOCIMIENTO, LIDERAZGO, DISEÑO E IMPLEMENTACIÓN DE LA ESTRATEGIA: UN ESTUDIO EMPÍRICO EN PEQUEÑAS Y MEDIANAS EMPRESAS. Ingeniare. Revista chilena de ingeniería, 18(3), 373-382. doi:10.4067/S071833052010000300011

Centro de Comercio Internacional. (2013). La Ventaja Competitiva Nacional. Forum de Comercio Internacional.

Esser, K. (1996). Competitividad Sistémica: Nuevo Desafío a las Empresas y a la Política. Revista de la CEPAL, 59, pág. 39 - 52.
Martín, I. (2007). Retos de la comunicación corporativa en la sociedad del conocimiento: de la gestión de información a la creación de conocimiento organizacional. Signo $y$ Pensamiento, 52-67.

Mejía, M., \& Colín, M. (2013). Gestióbn del conocimiento y su importancia en las organizaciones. Revista TRILOGÍA, 25-35. Obtenido de http://web.a.ebscohost.com/ehost/pdf viewer $/$ pdfviewer? vid $=5 \&$ sid $=872 \mathrm{c} 9$ bc1-e8ce-4a08-a0bcb005208c1bd8\%40sessionmgr4006

Nieves, Y. (2015). Activos de aprendizajer organizacional un activo de conocimiento critico . Ciencias de la Información , 31 - 37. Obtenido de https://www.redalyc.org/pdf/1814/18 1443340005.pdf

Passaillaigue, R., \& Estrada, V. (2016). La gestión del conocimiento y el aprendizaje organizacional en instituciones de educación superior. GECONTEC: Revista Internacional de Gestión del Conocimiento y la Tecnología, 35-43.

Peluffo, M., \& Catalán, E. (2002). Introducción a la gestión del conocimiento y su aplicación al sector publico. Santiago: Comisión Económica para América Latina y el Caribe (CEPAL). Obtenido de http://unpan1.un.org/intradoc/groups/ public/documents/uneclac/unpan0145 65.pdf

Pulido-Suárez, M., Ortiz-Zamora, A., \& Uribe, L. (2017). Las organizaciones en la economía del conocimiento:El papel fundamental de la gestión y transferencia del conocimiento en las organizaciones actuales. UniPluri/Versidad, 77-86. Obtenido de 


\section{LA COMUNICACIÓN COMO ESTRATEGIA DE DESARROLLO Y ORGANIZACIONAL EN LA GESTIÓN DEL CONOCIMIENTO}

Revista Economía y Administración, Vol. 9, No. 22018 ISSN 2219-6722 / ISSNE 2222-2707

http://web.a.ebscohost.com/ehost/pdf viewer/pdfviewer?vid $=3 \&$ sid $=872 \mathrm{c} 9$ bc1-e8ce-4a08-a0bcb005208c1bd8\%40sessionmgr4006

Sánchez, M. (2005). Breve inventario de los modelos para la gestión del conocimiento en las organizaciones. Acimed. Obtenido de http://eprints.rclis.org/7964/1/aci0606 05.pdf 